\title{
A GENERALIZATION OF THE SWARTZ EQUALITY
}

\author{
M. R. POURNAKI \\ Department of Mathematical Sciences, Sharif University of Technology, \\ P.O. Box 11155-9415, Tehran, Iran, and School of Mathematics, Institute for Research \\ in Fundamental Sciences (IPM), P.O. Box 19395-5746, Tehran, Iran \\ e-mail:pournaki@ipm.ir
}

\section{S. A. SEYED FAKHARI}

School of Mathematics, Institute for Research in Fundamental Sciences (IPM), P.O. Box 19395-5746, Tehran, Iran e-mail:fakhari@ipm.ir

and S. YASSEMI

School of Mathematics, Statistics and Computer Science, College of Science, University of Tehran, Tehran, Iran, and School of Mathematics, Institute for Research in Fundamental Sciences (IPM), P.O. Box 19395-5746,

Tehran, Iran

e-mail:yassemi@ipm.ir

(Received 11 October 2012; revised 9 April 2013; accepted 22 April 2013; first published online 30 August 2013)

\begin{abstract}
For a given $(d-1)$-dimensional simplicial complex $\Gamma$, we denote its $h$-vector by $h(\Gamma)=\left(h_{0}(\Gamma), h_{1}(\Gamma), \ldots, h_{d}(\Gamma)\right)$ and set $h_{-1}(\Gamma)=0$. The known Swartz equality implies that if $\Delta$ is a $(d-1)$-dimensional Buchsbaum simplicial complex over a field, then for every $0 \leq i \leq d$, the inequality $i h_{i}(\Delta)+(d-i+1) h_{i-1}(\Delta) \geq 0$ holds true. In this paper, by using these inequalities, we give a simple proof for a result of Terai (N. Terai, On $h$-vectors of Buchsbaum Stanley-Reisner rings, Hokkaido Math. $J$. 25(1) (1996), 137-148) on the $h$-vectors of Buchsbaum simplicial complexes. We then generalize the Swartz equality (E. Swartz, Lower bounds for $h$-vectors of $k$-CM, independence, and broken circuit complexes, SIAM J. Discrete Math. 18(3) (2004/05), 647-661), which in turn leads to a generalization of the above-mentioned inequalities for Cohen-Macaulay simplicial complexes in co-dimension $t$.
\end{abstract}

2000 Mathematics Subject Classification. Primary: 13F55, 05E45;

Secondary: $13 \mathrm{C} 14$

1. Introduction and preliminaries. Algebraic combinatorics is an area of mathematics that employs methods of abstract algebra in various combinatorial contexts and vice versa. One of the fastest developing subfields within algebraic combinatorics is combinatorial commutative algebra. It has evolved into one of the most active areas of mathematics during the past several decades. We refer the reader to the works of Bruns and Herzog [1] and Stanley [3] as general references on the subject.

Let us start with some preliminaries. A simplicial complex $\Delta$ on the set of vertices $[n]:=\{1, \ldots, n\}$ is a collection of subsets of $[n]$ which is closed under taking subsets; that 
is, if $F \in \Delta$ and $F^{\prime} \subseteq F$, then also $F^{\prime} \in \Delta$. Every element $F \in \Delta$ is called a face of $\Delta$, the size of a face $F$ is defined to be $|F|$ and its dimension is defined to be $|F|-1$. (As usual, for a given finite set $X$, the number of elements of $X$ is denoted by $|X|$.) The dimension of $\Delta$, which is denoted by $\operatorname{dim} \Delta$, is defined to be $d-1$, where $d=\max \{|F| \mid F \in \Delta\}$. A facet of $\Delta$ is a maximal face of $\Delta$ with respect to inclusion. We say that $\Delta$ is pure if all facets of $\Delta$ have the same cardinality. The link of $\Delta$ with respect to a face $F \in \Delta$, denoted by $\operatorname{lk}_{\Delta}(F)$, is the simplicial complex $\operatorname{lk}_{\Delta}(F)=\{G \subseteq[n] \backslash F \mid G \cup F \in \Delta\}$.

One of the connections between combinatorics and commutative algebra is via rings constructed from the combinatorial objects. Let $R=\mathbb{K}\left[x_{1}, \ldots, x_{n}\right]$ be the polynomial ring in $n$ variables over a field $\mathbb{K}$, and let $\Delta$ be a simplicial complex on $[n]$. For every subset $F \subseteq[n]$, we set $x_{F}=\prod_{i \in F} x_{i}$. The Stanley-Reisner ideal of $\Delta$ over $\mathbb{K}$ is the ideal $I_{\Delta}$ of $R$ which is generated by square-free monomials $x_{F}$ with $F \notin \Delta$. The Stanley-Reisner ring of $\Delta$ over $\mathbb{K}$, denoted by $\mathbb{K}[\Delta]$, is defined as $\mathbb{K}[\Delta]=R / I_{\Delta}$. A simplicial complex $\Delta$ is called Cohen-Macaulay over $\mathbb{K}$ (resp. Buchsbaum over $\mathbb{K}$ ), if its Stanley-Reisner ring $\mathbb{K}[\Delta]$ is a Cohen-Macaulay ring (resp. a Buchsbaum ring).

We now record the numerical data associated with a $(d-1)$-dimensional simplicial complex $\Delta$. Let $f_{i}(\Delta)$ denote the number of faces of $\Delta$ of dimension $i$. The sequence $f(\Delta)=\left(f_{0}(\Delta), f_{1}(\Delta), \ldots, f_{d-1}(\Delta)\right)$ is called the $f$-vector of $\Delta$. Also, to find out more about $f(\Delta)$, we need to study the $h$-vector of $\Delta$. Although it is seemingly complicated, often it is an elegant way to record the face numbers. Letting $f_{-1}(\Delta)=1$, we define the $h$-vector $h(\Delta)=\left(h_{0}(\Delta), h_{1}(\Delta), \ldots, h_{d}(\Delta)\right)$ of $\Delta$ by the formula

$$
\sum_{i=0}^{d} h_{i}(\Delta) t^{d-i}=\sum_{i=0}^{d} f_{i-1}(\Delta)(t-1)^{d-i}
$$

and for every $i<0$, we set $h_{i}(\Delta)=0$. One can easily check that

$$
f_{i-1}(\Delta)=\sum_{j=0}^{i}\left(\begin{array}{l}
d-j \\
i-j
\end{array}\right) h_{j}(\Delta)
$$

and

$$
h_{i}(\Delta)=\sum_{j=0}^{i}(-1)^{i-j}\left(\begin{array}{l}
d-j \\
i-j
\end{array}\right) f_{j-1}(\Delta) .
$$

It is well known that

$$
H_{\mathbb{K}[\Delta]}(t)=\frac{\sum_{i=0}^{d} h_{i}(\Delta) t^{i}}{(1-t)^{d}},
$$

where $H_{\llbracket \ll[\Delta]}(t)$ is the Hilbert series of $\mathbb{K}[\Delta]$ and $d=\operatorname{dim} \Delta+1$ is its Krull dimension.

The study of $h$-vectors of simplicial complexes has long been a topic of interest both in combinatorics and combinatorial commutative algebra. In this direction, let us state an equality, called the Swartz equality. 
Proposition 1.1 ([4], Proposition 2.3). Let $\Delta$ be a pure $(d-1)$-dimensional simplicial complex. Then for every $0 \leq i \leq d$, we have

$$
i h_{i}(\Delta)+(d-i+1) h_{i-1}(\Delta)=\sum_{v \in[n]} h_{i-1}\left(\mathrm{lk}_{\Delta}(v)\right) .
$$

It is well known that $\Delta$ is Buchsbaum over a field $\mathbb{K}$ if and only if it is pure and for every non-empty face $F$ of $\Delta$, the link of $\Delta$ with respect to $F$ is Cohen-Macaulay over $\mathbb{K}($ see [3, Theorem 8.1, p. 73]). Also, by a classical result of Stanley, if $\Delta$ is CohenMacaulay over $\mathbb{K}$ with dimension $d-1$, then for every $0 \leq i \leq d$, we have $h_{i}(\Delta) \geq 0$ (see [3, Theorem 3.3, p. 59]). By combining these facts with Proposition 1.1, the Swartz equality, we conclude the following corollary.

COROLLARY 1.2. Let $\Delta$ be a $(d-1)$-dimensional Buchsbaum simplicial complex over a field $\mathbb{K}$. Then for every $0 \leq i \leq d$, we have

$$
i h_{i}(\Delta)+(d-i+1) h_{i-1}(\Delta) \geq 0 .
$$

Terai in [5] has proved some inequalities for the $h$-vectors of Buchsbaum simplicial complexes. In Proposition 2.1, by using Corollary 1.2, we give a simple proof for Terai's result, which shows that this corollary is stronger than his result. This is a motivation for us to see whether we can extend Corollary 1.2 for a larger class of simplicial complexes than that of Buchsbaum. Recently, Haghighi et al. [2] have defined the notion of $\mathrm{CM}_{t}$ simplicial complexes as a generalization of Cohen-Macaulay and Buchsbaum simplicial complexes (see Definition 2.2). This notion is different from that of the so-called $t$-CM, which may be found in, for example, [4]. The notion of $\mathrm{CM}_{0}$ simplicial complexes agrees with the notion of Cohen-Macaulay simplicial complexes, and the notion of $\mathrm{CM}_{1}$ simplicial complexes agrees with that of Buchsbaum simplicial complexes. In Proposition 2.4, we give a generalization of Corollary 1.2 for $\mathrm{CM}_{t}$ simplicial complexes. In order to do this, we need to generalize Proposition 1.1, the Swartz equality, which we state and prove in Theorem 2.3.

2. The results. We start this section by stating the following proposition, which has been already proved by Terai [5]. Here we give a simple proof for this proposition by using Corollary 1.2.

Proposition 2.1 ([5], Theorem 2.1). Let $\mathbb{K}$ be a field and $\Delta$ be a $(d-1)$-dimensional Buchsbaum simplicial complex over $\mathbb{K}$. Then the following inequalities hold true.

(1) $d h_{d}(\Delta)+h_{d-1}(\Delta) \geq 0$,

(2) $\left(\begin{array}{l}d \\ 2\end{array}\right) h_{d}(\Delta)+(d-1) h_{d-1}(\Delta)+h_{d-2}(\Delta) \geq 0$,

(3) $\left(\begin{array}{l}d \\ 3\end{array}\right) h_{d}(\Delta)+\left(\begin{array}{c}d-1 \\ 2\end{array}\right) h_{d-1}(\Delta)+(d-2) h_{d-2}(\Delta)+h_{d-3}(\Delta) \geq 0$,

(4) $\sum_{k=0}^{i}\left(\begin{array}{c}d+i-k-3 \\ i-k\end{array}\right) h_{d-k}(\Delta) \geq 0$, if $i \geq 4$. 
Proof. By Corollary 1.2, for every $0 \leq i \leq d$, we have $i h_{i}(\Delta)+(d-i+1) h_{i-1}(\Delta) \geq$ 0 . This implies that for every $1 \leq i \leq d$, we have

$$
\begin{aligned}
0 \leq & \frac{1}{i} \sum_{k=1}^{i}\left(\begin{array}{c}
d-k \\
i-k
\end{array}\right)\left((d-k+1) h_{d-k+1}(\Delta)+k h_{d-k}(\Delta)\right) \\
= & \frac{d}{i}\left(\begin{array}{c}
d-1 \\
i-1
\end{array}\right) h_{d}(\Delta)+\frac{1}{i} \sum_{k=1}^{i-1}\left\{k\left(\begin{array}{c}
d-k \\
i-k
\end{array}\right)+(d-k)\left(\begin{array}{c}
d-k-1 \\
i-k-1
\end{array}\right)\right\} h_{d-k}(\Delta) \\
& +h_{d-i}(\Delta),
\end{aligned}
$$

and so we conclude that

$$
\sum_{k=0}^{i}\left(\begin{array}{l}
d-k \\
i-k
\end{array}\right) h_{d-k}(\Delta) \geq 0
$$

Now the proof of (1), (2) and (3) follows from inequality ( $\dagger$ ) by substituting $i=1$, $i=2$ and $i=3$ respectively. For proof of (4), by inequality ( $\dagger$ ), for every $i \geq 4$, we have

$$
\begin{aligned}
0 \leq & \sum_{j=3}^{i} \sum_{k=0}^{j}\left(\begin{array}{c}
i-3 \\
i-j
\end{array}\right)\left(\begin{array}{l}
d-k \\
j-k
\end{array}\right) h_{d-k}(\Delta) \\
= & \sum_{j=3}^{i}\left(\begin{array}{l}
i-3 \\
i-j
\end{array}\right)\left(\begin{array}{l}
d \\
j
\end{array}\right) h_{d}(\Delta)+\sum_{j=3}^{i}\left(\begin{array}{c}
i-3 \\
i-j
\end{array}\right)\left(\begin{array}{c}
d-1 \\
j-1
\end{array}\right) h_{d-1}(\Delta) \\
& +\sum_{j=3}^{i}\left(\begin{array}{c}
i-3 \\
i-j
\end{array}\right)\left(\begin{array}{c}
d-2 \\
j-2
\end{array}\right) h_{d-2}(\Delta)+\sum_{k=3}^{i} \sum_{j=k}^{i}\left(\begin{array}{c}
i-3 \\
i-j
\end{array}\right)\left(\begin{array}{c}
d-k \\
j-k
\end{array}\right) h_{d-k}(\Delta) \\
= & \left(\begin{array}{c}
d+i-3 \\
i
\end{array}\right) h_{d}(\Delta)+\left(\begin{array}{c}
d+i-4 \\
i-1
\end{array}\right) h_{d-1}(\Delta)+\left(\begin{array}{c}
d+i-5 \\
i-2
\end{array}\right) h_{d-2}(\Delta) \\
& +\sum_{k=3}^{i}\left(\begin{array}{c}
d+i-k-3 \\
i-k
\end{array}\right) h_{d-k}(\Delta) \\
= & \sum_{k=0}^{i}\left(\begin{array}{c}
d+i-k-3 \\
i-k
\end{array}\right) h_{d-k}(\Delta),
\end{aligned}
$$

as required.

Let us define the notion of $\mathrm{CM}_{t}$ simplicial complexes.

DEFINITION 2.2. Let $t$ be a non-negative integer, $\mathbb{K}$ a field and $\Delta$ a pure simplicial complex. We say that $\Delta$ is a $\mathrm{CM}_{t}$ simplicial complex over $\mathbb{K}$ if for every $F \in \Delta$ with $|F| \geq t$, the link of $\Delta$ with respect to $F$ is Cohen-Macaulay over $\mathbb{K}$.

We now generalize Corollary 1.2 for $\mathrm{CM}_{t}$ complexes. In order to do this, we state and prove the following theorem, which is a generalization of Proposition 1.1, the Swartz equality. 
THEOREM 2.3. Let $\Delta$ be a pure $(d-1)$-dimensional simplicial complex. Then for every $1 \leq t \leq d$ and every $0 \leq i \leq d$, we have

$$
\sum_{\substack{F \in \Delta \\
|F|=t}} h_{i-t}\left(\mathrm{lk}_{\Delta}(F)\right)=\sum_{k=0}^{t}\left(\begin{array}{c}
i-k \\
t-k
\end{array}\right)\left(\begin{array}{c}
d-i+k \\
k
\end{array}\right) h_{i-k}(\Delta) .
$$

Proof. We prove the theorem by induction on $t$. The case $t=1$ follows from Proposition 1.1. We now assume that $t \geq 2$. Suppose that the assertion is true for $t-1$. Therefore, we have

$$
\sum_{\substack{F \in \Delta \\
|F|=t-1}} h_{i-t+1}\left(\mathrm{lk}_{\Delta}(F)\right)=\sum_{k=0}^{t-1}\left(\begin{array}{c}
i-k \\
t-k-1
\end{array}\right)\left(\begin{array}{c}
d-i+k \\
k
\end{array}\right) h_{i-k}(\Delta) .
$$

By using Proposition 1.1, we conclude that

$$
\begin{aligned}
\sum_{\substack{F \in \Delta \\
|F|=t}} h_{i-t}\left(\mathrm{lk}_{\Delta}(F)\right)= & \frac{1}{t} \sum_{\substack{F \in \Delta \\
|F|=t-1}} \sum_{u \in \mathrm{k}_{\Delta}(F)} h_{i-t}\left(\mathrm{lk}_{\mathrm{lk}_{\Delta}(F)}(u)\right) \\
= & \frac{1}{t} \sum_{\substack{F \in \Delta \\
|F|=t-1}}\left\{(i-t+1) h_{i-t+1}\left(\mathrm{lk}_{\Delta}(F)\right)\right. \\
& \left.+(d-t+1-i+t) h_{i-t}\left(\mathrm{lk}_{\Delta}(F)\right)\right\} \\
= & \frac{1}{t}(i-t+1) \sum_{\substack{F \in \Delta \\
|F|=t-1}} h_{i-t+1}\left(\operatorname{lk}_{\Delta}(F)\right) \\
& +\frac{1}{t}(d-i+1) \sum_{\substack{F \in \Delta \\
|F|=t-1}} h_{i-t}\left(1 \mathrm{k}_{\Delta}(F)\right) .
\end{aligned}
$$

Now, the induction hypothesis implies that

$$
\begin{aligned}
\sum_{\substack{F \in \Delta \\
|F|=t}} h_{i-t}\left(\mathrm{lk}_{\Delta}(F)\right)= & \frac{1}{t}(i-t+1) \sum_{k=0}^{t-1}\left(\begin{array}{c}
i-k \\
t-k-1
\end{array}\right)\left(\begin{array}{c}
d-i+k \\
k
\end{array}\right) h_{i-k}(\Delta) \\
& +\frac{1}{t}(d-i+1) \sum_{k=0}^{t-1}\left(\begin{array}{c}
i-k-1 \\
t-k-1
\end{array}\right)\left(\begin{array}{c}
d-i+k+1 \\
k
\end{array}\right) h_{i-k-1}(\Delta) \\
= & \left(\begin{array}{c}
i \\
t
\end{array}\right) h_{i}(\Delta)+\sum_{k=1}^{t-1}\left\{\frac{1}{t}(i-t+1)\left(\begin{array}{c}
i-k \\
t-k-1
\end{array}\right)\left(\begin{array}{c}
d-i+k \\
k
\end{array}\right)\right. \\
& \left.+\frac{1}{t}(d-i+1)\left(\begin{array}{c}
i-k \\
t-k
\end{array}\right)\left(\begin{array}{c}
d-i+k \\
k-1
\end{array}\right)\right\} h_{i-k}(\Delta)+\left(\begin{array}{c}
d-i+t \\
t
\end{array}\right) h_{i-t}(\Delta)
\end{aligned}
$$




$$
\begin{aligned}
= & \left(\begin{array}{c}
i \\
t
\end{array}\right) h_{i}(\Delta)+\sum_{k=1}^{t-1}\left(\begin{array}{c}
i-k \\
t-k
\end{array}\right)\left(\begin{array}{c}
d-i+k \\
k
\end{array}\right) h_{i-k}(\Delta) \\
& +\left(\begin{array}{c}
d-i+t \\
t
\end{array}\right) h_{i-t}(\Delta) \\
= & \sum_{k=0}^{t}\left(\begin{array}{c}
i-k \\
t-k
\end{array}\right)\left(\begin{array}{c}
d-i+k \\
k
\end{array}\right) h_{i-k}(\Delta),
\end{aligned}
$$

as required.

Since every component of the $h$-vector of a Cohen-Macaulay simplicial complex over a field $\mathbb{K}$ is non-negative, Theorem 2.3 implies the following generalization of Corollary 1.2.

Proposition 2.4. Let $t$ be a non-negative integer and $\mathbb{K}$ a field. Let $\Delta$ be a $(d-1)-$ dimensional $\mathrm{CM}_{t}$ simplicial complex over $\mathbb{K}$. Then for every $0 \leq i \leq d$, we have

$$
\sum_{k=0}^{t}\left(\begin{array}{c}
i-k \\
t-k
\end{array}\right)\left(\begin{array}{c}
d-i+k \\
k
\end{array}\right) h_{i-k}(\Delta) \geq 0 .
$$

Acknowledgements. The research of M. R. Pournaki, S. A. Seyed Fakhari and S. Yassemi was in part supported by a grant from IPM (Nos. 91130115, 92130422 and 91130214). This work has been also partially supported by the Center for International Scientific Studies and Collaboration (CISSC), Iran, and Égide - Programme Gundishapur 2012, Hubert Curien Partnership, France (No. 27462PL). The authors would like to thank the referee for the comments and suggestions.

\section{REFERENCES}

1. W. Bruns and J. Herzog, Cohen-Macaulay rings, Cambridge Studies in Advanced Mathematics, 39 (Cambridge University Press, Cambridge, UK, 1993).

2. H. Haghighi, S. Yassemi and R. Zaare-Nahandi, A generalization of $k$-CohenMacaulay simplicial complexes, Ark. Mat. 50(2) (2012), 279-290.

3. R. P. Stanley, Combinatorics and commutative algebra, 2nd ed., Progress in Mathematics, 41 (Birkhäuser, Boston, MA, 1996).

4. E. Swartz, Lower bounds for $h$-vectors of $k$-CM, independence, and broken circuit complexes, SIAM J. Discrete Math. 18(3) (2004/05), 647-661.

5. N. Terai, On $h$-vectors of Buchsbaum Stanley-Reisner rings, Hokkaido Math. J. 25(1) (1996), 137-148. 